\title{
ANTIBACTERIAL ACTIVITY OF AQUEOUS GARLIC EXTRACT ON BETA-LACTAMASE- PRODUCING ACINETOBACTER ISOLATES FROM SKIN AND SOFT TISSUE INFECTIONS
}

\author{
PRATIBHA J SHAH ${ }^{1 *}$, MANITA T WILLIAMSON ${ }^{2}$, SEJAL J RATHOD ${ }^{1}$
}

\author{
${ }^{1}$ Department of Microbiology, K.C. College, Churchgate, Mumbai, Maharashtra, India. ${ }^{2}$ Department of Microbiology, T.N.M.C \& B.Y.L. Nair \\ Charitable Hospital, Mumbai, India. Email: shahpratij@gmail.com
}

Received: 07 July 2016, Revised and Accepted: 28 July 2016

\section{ABSTRACT}

Objective: The present study evaluates the antibacterial effect of aqueous garlic extract (AGE) on beta-lactamase-producing Acinetobacter strains isolated from skin and soft tissue infections (SSTIs).

Methods: A total of 41 non-duplicate strains of Acinetobacter isolated from SSTIs specimens were tested for their antibiotic susceptibility pattern by Kirby-Bauer disk diffusion method. Ceftazidime-resistant Acinetobacter strains were screened for beta-lactamase production by phenotypic confirmatory disc diffusion test and E-test. Antibacterial activity of AGE was examined by the disc diffusion method, and the minimum inhibitory concentration (MIC) of AGE and cefotaxime was determined by the agar dilution technique. The combined activity of AGE and cefotaxime was evaluated by calculating the fractional inhibitory concentration index by the checkerboard method.

Results: A total of 21 Acinetobacter strains were confirmed to be beta-lactamase producers, out of which, 6 were metallo-beta-lactamase producers, 3 were Ambler Class C, and 12 were multiple beta-lactamase producers. AGE exhibited a significant antibacterial activity as the observed zones of inhibition ranged from 18 to $31 \mathrm{~mm}$ against the test strains. The MIC of AGE was in the range of $0.5-2 \%$ (2.5-10 mg/ml) with a mean of $0.86 \%$ $(4.28 \mathrm{mg} / \mathrm{ml})$. About 11 test strains showed synergism, 5 strains exhibited additive and indifferent effect, each. By high-performance liquid chromatography analysis, the concentration of allicin was found to be 0.20 , expressed as percentage $\mathrm{w} / \mathrm{w}$.

Conclusion: It can be stated that AGE might have therapeutic value against beta-lactamase-producing Acinetobacter isolates from SSTIs.

Keywords: Beta-lactamase, Aqueous garlic extract, Skin and soft tissue infections, Allicin, Synergy.

(C) 2016 The Authors. Published by Innovare Academic Sciences Pvt Ltd. This is an open access article under the CC BY license (http://creativecommons. org/licenses/by/4. 0/) DOI: http://dx.doi.org/10.22159/ajpcr.2016.v9i6.13960

\section{INTRODUCTION}

During the past decade, Acinetobacter species have emerged as an opportunistic pathogen. They are rapidly evolving toward multidrug resistance and are often involved in various nosocomial infections that can be severe in intensive care units, burn units, and surgical wards [1] Multidrug-resistant strains of Acinetobacter generally cause treatment failure as therapeutic options are limited for infections caused by them [2]

Skin or tissue infections, skin structure, and deep-seated soft tissue infections are general terms used for infections of the entire skin layer, including the subcutaneous and muscle tissue layers and their respective fascia structures. The beta-lactam antibiotics are the most commonly used drug for the treatment of various skin and soft tissue infections (SSTIs). The resistance against beta-lactam is swiftly increasing in Acinetobacter strains [3]. A variety of molecular mechanisms for resistance to broad-spectrum beta-lactams have been reported in Acinetobacter, such as mutations of penicillin-binding proteins and alterations of membrane permeability, but the most common mechanism is attributed to the presence of betalactamases encoded by either chromosomes or plasmids [4].

Extended-spectrum beta-lactamases (ESBLs) are typically plasmidmediated clavulanate-susceptible enzymes that hydrolyze penicillins, expanded-spectrum cephalosporins, monobactams, and are commonly inhibited by beta-lactamase inhibitors such as clavulanic acid, sulbactam, and tazobactam [5]. Ambler Class C (AmpC) class betalactamases are cephalosporinases that are poorly inhibited by clavulanic acid. They can be differentiated from ESBLs by their ability to hydrolyze cephamycins as well as other extended-spectrum cephalosporins [6] Metallo-beta-lactamases (MBLs) are a type of carbapenemase, which are characterized by the ability to hydrolyze carbapenems and are inhibited by ethylenediaminetetraacetic acid (EDTA), chelators of $\mathrm{Zn}^{2+}$. They lack inhibition by the commercially available beta-lactam inhibitors; clavulanic acid, sulbactam, and tazobactam [7]. Often coexistence of multiple beta-lactamase enzymes in a single isolate is observed, which further complicates treatment options [8].

There is a lack of development of new synthetic antimicrobials against Gram-negative organisms, hence attention is increasingly focused on natural compounds either as stand-alone or adjunctive therapy [9] Plants produce a wide variety of secondary metabolites, which are used either directly as precursors or as compounds in the pharmaceutical industry [10].

Garlic (Allium sativum) has been found to exhibit a wide spectrum of antibacterial activity against Gram-negative and Gram-positive bacteria, including species of Escherichia, Salmonella, Staphylococcus, Streptococcus, Klebsiella, Proteus, Bacillus, and Clostridium [11] Cavallito and Bailey were the first to demonstrate that the antibacterial action of garlic is mainly due to allicin [12]. Allicin is generated by the enzyme alliinase when garlic is crushed. Garlic contains at least 33 sulfur compounds such as alliin, allicin, ajoene, allyl propyl, diallyl trisulfide, s-allyl cysteine, S-allylmercaptocysteine, and others, which are responsible for its antibacterial activity [13]

Allicin interacts with important thiol-containing enzymes such as cysteine proteinases, alcohol dehydrogenase, as well as the thioredoxin reductases, which are critical for maintaining the correct redox state within microorganisms. It is unlikely that bacteria would develop resistance to allicin because they would require modifying these vital enzymes, which are necessary for the survival of the microbes. Hence, garlic may prove to be a potent antimicrobial agent [14]. 
Very few studies have been documented on the antibacterial activity of aqueous garlic extract (AGE) against beta-lactamase producers. No study has reported the effect of AGE against beta-lactamase-producing Acinetobacter strains from SSTIs. Thus, this study aims to investigate the antibacterial activity of AGE on beta-lactamase-producing Acinetobacter strains isolated from SSTIs and also to study its combined activity along with cefotaxime.

\section{METHODS}

The present study was conducted in our tertiary care hospital and was approved by the Local Ethics Committee of the institution.

\section{Bacterial strains}

A total of 41 non-duplicate Acinetobacter strains isolated from SSTIs of patients from our tertiary care hospital were selected for the study. The samples were processed and were identified by standard laboratory methods [15].

\section{Antimicrobial susceptibility test (AST)}

The antimicrobial susceptibility was determined by Kirby-Bauer disk diffusion method in accordance with the Clinical and Laboratory Standards Institute (CLSI) guidelines using commercially available antimicrobial discs (HiMedia, Mumbai, India) [16]. The following antibiotics were used-ampicillin $(10 \mu \mathrm{g})$, amikacin $(10 \mu \mathrm{g})$, ciprofloxacin (5 $\mu \mathrm{g})$, gentamicin $(10 \mu \mathrm{g})$, amoxyclav $(30 \mu \mathrm{g})$, ceftazidime $(30 \mu \mathrm{g})$, cefoxitin $(30 \mu \mathrm{g})$, imipenem $(10 \mu \mathrm{g})$, meropenem $(10 \mu \mathrm{g})$, piperacillin $(10 \mu \mathrm{g})$, and piperacillin-tazobactam $(100 / 10)$.

All the isolates which were resistant to ceftazidime, as per the CLSIsusceptible breakpoints, were further screened for beta-lactamase production by the confirmatory tests.

\section{Phenotypic confirmatory disc diffusion test (PCDDT)}

The phenotypic disc diffusion test was performed as per the CLSI guidelines. The current CLSI guideline does not describe any method for the detection of isolates producing AmpC beta-lactamases. The antibiotic discs were purchased from HiMedia Laboratories, Mumbai, India.

Ceftazidime $(30 \mu \mathrm{g})$ - ceftazidime/clavulanic acid (30/10) was used for ESBL detection. If there was $\geq 5 \mathrm{~mm}$ increase in the inhibition zone diameter of ceftazidime/clavulanic acid versus ceftazidime alone, the isolate was considered an ESBL producer [16]. Imipenem/ EDTA $(10 / 750 \mu \mathrm{g})$ disc was used for the detection of MBL producers. An increase of $\geq 7 \mathrm{~mm}$ in the inhibition zone diameter of imipenem/ EDTA versus imipenem alone indicated MBL production [16]. Cefoxitin $(30 \mu \mathrm{g})$ - cefoxitin/cloxacillin (30/200) discs was used for AmpC detection. An increase of $\geq 4 \mathrm{~mm}$ in the inhibition zone diameter of cefoxitin/cloxacillin acid versus cefoxitin alone indicated AmpC production [17].

\section{E-test}

Detection by phenotypic testing may be misleading, especially when there is a coexistence of multiple beta-lactamase enzymes. They mask each other, which results in misreporting and failure in the clinical treatment of patients. For this reason, E-strips which differ from conventional strips have been used. Different inhibitors have been applied to improvise the phenotypic tests for beta-lactamase detection.

ESBL and AmpC detection Ezy MIC ${ }^{\text {Tm }}$ strip (EM081 - HiMedia, Mumbai, India) are drug-impregnated strips, in which the upper half contains a concentration gradient of 4 antibiotics; ceftazidime, cefotaxime, cefepime and cloxacillin plus clavulanic acid, and tazobactam and the lower half contains ceftazidime, cefotaxime, cefepime, and cloxacillin in a concentration gradient in a reverse direction. The isolates were reported and confirmed as ESBL and AmpC beta-lactamase producer as per the application sheet supplied by the manufacturer. These strips are to be used along with pure ESBL detection strips (EM079-HiMedia, Mumbai, India) to avoid false-positive results [16-18].
The phenotypic MBL detection strip (EM078 - HiMedia, Mumbai, India) is coated with a mixture of imipenem+EDTA and imipenem on a single strip in a concentration gradient manner. The upper half has imipenem+EDTA with highest concentration tapering downward, whereas the lower half is similarly coated with imipenem in a concentration gradient in the reverse direction. The isolates were reported and confirmed as MBL producer when the ratio of the value obtained for imipenem and the value of imipenem+EDTA was $>8[16,19]$.

A standard reference strain of Escherichia coli ATCC 25922, susceptible to all antimicrobial drugs tested, and positive control strain Klebsiella pneumoniae ATCC 700603 were used as a quality control for AST, PCDDT, and the E-test, as per the CLSI guidelines. These phenotypically confirmed ESBL, AmpC, and MBL producers were further utilized in the study as test strains.

\section{Preparation of AGE}

The garlic cloves were peeled, cut into pieces, and $20 \mathrm{~g}$ was put in a juice extractor to squeeze out the raw garlic extract. This extract was regarded as $100 \%$ extract and used for further studies. To achieve various concentrations, the extract was diluted with sterilized distilled water. To estimate the dry weight of the extract, $10 \mathrm{ml}$ of the extract was placed in a pre-weighed glass dish and re-weighed, followed by drying in an oven at $60^{\circ} \mathrm{C}$. The sample was found to contain $5 \mathrm{~g}$ (equivalent dry weight) of garlic extract in $10 \mathrm{ml}$, which was equivalent to $500 \mathrm{mg} / \mathrm{ml}$ and was used as a stock AGE [20]. This extract was then stored at $4{ }^{\circ} \mathrm{C}$ for further use.

\section{High-performance liquid chromatography analysis (HPLC)}

The HPLC system consisted of a stainless steel column $(0.25 \mathrm{~m}$ long and $4 \mathrm{~mm}$ in internal diameter, packed with silanised octadecylsilyl silica gel) combined with a stainless steel pre-column $(20 \mathrm{~mm}$ long and $4 \mathrm{~mm}$ in internal diameter packed with silanised octadecylsilyl silica gel). The elution was carried out at a flow rate of $0.8 \mathrm{ml} / \mathrm{min}$ using mobile phase made up of a mixture of 40 volumes of a $1 \% \mathrm{v} / \mathrm{v}$ solution of anhydrous formic acid and 60 volumes of methanol. About $20.0 \mathrm{mg}$ of butyl parahydroxybenzoate was dissolved in $100.0 \mathrm{ml}$ of a mixture of equal volumes of methanol and water and was used as an internal standard. Detection was performed at a wavelength of $254 \mathrm{~nm}$. About $0.800 \mathrm{~g}$ of garlic powder was homogenized in $20.0 \mathrm{ml}$ of water, and $10 \mu \mathrm{l}$ was injected into the HPLC (Natural Remedies, Bangalore, India). The percentage of allicin in the sample was calculated using the following formula:

\section{$\mathrm{S} 1 \times \mathrm{m} 2 \times 22.75$ \\ $\mathrm{S} 2 \times \mathrm{m} 1$}

S1=Area of the peak corresponding to allicin,

S2=Area of the peak corresponding to butyl parahydroxybenzoate in the chromatogram obtained with the test solution,

$\mathrm{m} 1=$ Mass of the drug in grams,

$\mathrm{m} 2=$ Mass of butyl parahydroxybenzoate in grams in $100.0 \mathrm{ml}$ of the internal standard solution.

$1 \mathrm{mg}$ of butyl parahydroxybenzoate corresponds to $8.65 \mathrm{mg}$ of allicin

\section{Antibacterial activity of AGE}

The evaluation of antibacterial activity of AGE was conducted by the disc diffusion method using Mueller-Hinton agar as described by the CLSI [16]. The turbidity of the culture was adjusted to 0.5 McFarland standards. Sterile paper discs ( $6 \mathrm{~mm}$, HiMedia, Mumbai, Maharashtra, India) were impregnated with $20 \mu \mathrm{l}$ of the $500 \mathrm{mg} / \mathrm{ml} \mathrm{AGE}$ and placed on the inoculated agar. For the positive control, a disc of imipenem $(10 \mu \mathrm{g})$, and for negative control, a disc impregnated with distilled water was placed on the inoculated Mueller-Hinton agar. The plate was incubated at $37^{\circ} \mathrm{C}$ for $24 \mathrm{hr}$. The experiment was performed in triplicate 
Determination of minimum inhibitory concentration (MIC) The MIC of AGE and cefotaxime was determined by agar dilution method [21]. For MIC of AGE, dilutions were prepared by mixing AGE with sterile Mueller-Hinton agar to get final concentrations ranging between $0.25-4 \%$ (1.25-20 mg/ml). For MIC of cefotaxime, dilutions were prepared by mixing cefotaxime with sterile Mueller-Hinton Agar to get the final concentrations ranging from 25 to $500 \mu \mathrm{g} / \mathrm{ml}$. A plate of Mueller-Hinton agar without AGE served as a control. These plates were seeded with bacterial suspensions and were incubated at $37^{\circ} \mathrm{C}$ for $24 \mathrm{hr}$. The MIC was recorded as the lowest concentration of AGE and cefotaxime, at which visible bacterial growth was completely inhibited.

Determination of synergistic activity by checkerboard assay using Agar dilution method

Checkerboard assay was used to establish the presence of synergistic interaction between cefotaxime (stock $-10 \mathrm{mg} / \mathrm{ml}$ ) and AGE (10\%). The agar plates were prepared by mixing AGE in the range of $0.1-2 \%(0.5-10 \mathrm{mg} / \mathrm{ml})$ along with $10-200 \mu \mathrm{g} / \mathrm{ml}$ of cefotaxime. Visible growth after $24 \mathrm{hr}$ of incubation at $37^{\circ} \mathrm{C}$ was checked, and the MIC in combination with cefotaxime and AGE was determined for all Acinetobacter strains. The results obtained were used to calculate fractional inhibitory concentration (FIC) indices. The FIC index ( $\sum$ FIC) was calculated as follows:

\section{$\sum$ FIC $=$ FIC A+FICB}

FIC A=MIC of cefotaxime in combination/MIC of cefotaxime alone

\section{FIC B=MIC of AGE in combination/MIC of AGE alone}

A minimum FIC index of $\leq 0.5$ indicates synergy, while a FIC index $>2$ indicates antagonism. If the minimum FIC index was $>0.5$ and $\leq 1$, the effect of the combination was classified as additive. If the minimum FIC index was $>1$ and $\leq 2$, the effect of the combination was classified as indifference [21].

\section{RESULTS}

The Acinetobacter strains isolated from SSTIs showed the highest rate of resistance against ciprofloxacin and ampicillin, $80 \%$ each $(33 / 41)$. The lowest rate of resistance was exhibited against amikacin (17\%). Among all the isolates, $63.4 \%(26 / 41)$ strains were resistant to ceftazidime whereas $21.95 \%$ (9/41) strains were resistant to carbapenems.

Resistance to a third-generation cephalosporin, ceftazidime, was indicative of beta-lactamase production, which was further confirmed by PCDDT and E-test. Nearly, $51.2 \%$ of the $(21 / 41)$ strains were confirmed to be beta-lactamase producers, and these were used as test organisms further in the study. Among all the test strains, 7.31\% (3/41) strains were detected to be pure AmpC producers. There were no pure ESBL producers, but $29.3 \%$ (12/41) of the strains were co-producers of ESBL and AmpC, i.e., they were multiple beta-lactamase producers. Out of the 9 strains resistant to imipenem, 6 strains were MBL producers. Thus, $15 \%$ of the isolates were MBL producers (6/41). Out of the 12 co-producers of ESBL and AmpC, 3 were reported to be sensitive to piperacillin-tazobactam combination, in vitro. All beta-lactamase producers were also found to be multidrug-resistant, i.e., they were resistant to 3 or more than 3 groups of antibiotics.

The estimation of active ingredient allicin was carried out by HPLC analysis and was found to be 0.20 , expressed as percentage $w / w$. Antibacterial activity of AGE was carried out by the disc diffusion method, and the average zone of inhibition ranged from 18 to $31 \mathrm{~mm}$ with a mean of $25.19 \pm 5.06 \mathrm{~mm}$ against the test strains (Fig. 1).

The MIC of AGE and cefotaxime was determined by the agar dilution method. The MIC of AGE was found to be in the range of $0.5-2 \%(2.5-10 \mathrm{mg} / \mathrm{ml})$ with a mean of $0.86 \%(4.28 \pm 2.25 \mathrm{mg} / \mathrm{ml})$ as shown in Table 1. The MIC of cefotaxime was found to be in the range of $100-400 \mu \mathrm{g} / \mathrm{ml}$ with an average of $252.38 \mu \mathrm{g} / \mathrm{ml}$ (Table 2).
Table 1 depicts the number and percentage of Acinetobacter strains inhibited by various minimal concentrations of AGE by agar dilution method $(\mathrm{n}=21)$.

Table 2 depicts the number and percentage of Acinetobacter strains inhibited by various concentrations of cefotaxime by agar dilution method $(n=21)$.

Checkerboard assay was used to determine the combined activity of various concentrations of cefotaxime and AGE. FIC index was used to determine the type of association between cefotaxime and AGE. Based on the FIC index, 11 test strains showed synergism, 5 strains exhibited additive and indifference effect each. None of the strains showed an antagonistic effect between AGE and cefotaxime (Table 3).

Table 3 depicts the number of the Acinetobacter strains inhibited by combining the concentrations of AGE and cefotaxime $(n=21)$. Synergistic interaction between AGE and cefotaxime was seen in $52.38 \%$ of the isolates, and none of the isolates exhibited antagonism.

\section{DISCUSSION}

Multidrug-resistant Acinetobacter strains have been identified to be among the most difficult antimicrobial-resistant Gram-negative bacilli to control and treat. The study of the pattern of bacterial resistance is important for epidemiological and clinical purposes. In the current study, amikacin was the most effective antibiotic for Acinetobacter isolates, followed by imipenem. The resistant rate in Acinetobacter strains toward ceftazidime and imipenem was in accordance with the study carried out by Parandekar and Peerapur at a hospital in Pune [22].

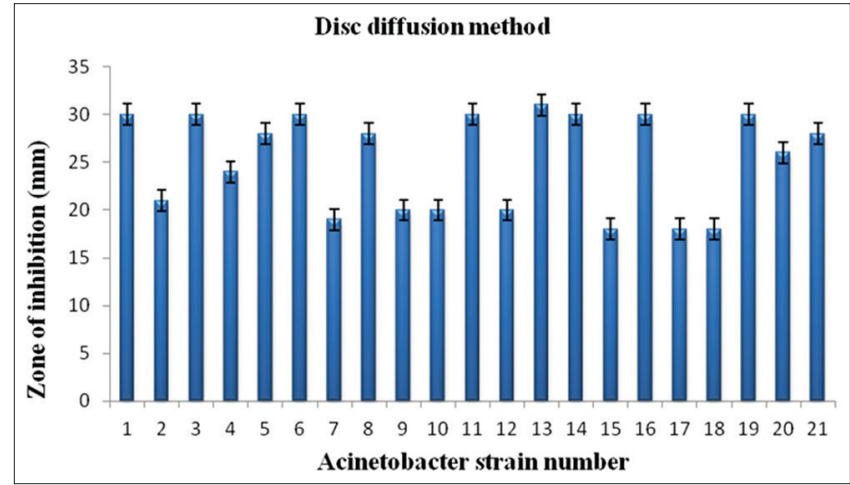

Fig. 1: Bar chart showing mean inhibition zone sizes of aqueous garlic extract by disc diffusion method (Data are expressed as mean \pm standard error of mean, $n=3$ )

Table 1: MIC of AGE against Acinetobacter strains producing beta-lactamase

\begin{tabular}{llllll}
\hline $\begin{array}{l}\text { Concentration of AGE } \\
\text { (mg/ml) }\end{array}$ & $\mathbf{1 . 2 5}$ & $\mathbf{2 . 5}$ & $\mathbf{5}$ & $\mathbf{1 0}$ & $\mathbf{2 0}$ \\
\hline $\begin{array}{l}\text { Number of strains inhibited } \\
\text { Percentage of strains inhibited }\end{array}$ & - & 10 & 9 & 2 & - \\
\hline
\end{tabular}

MIC: Minimum inhibitory concentration, AGE: Aqueous garlic extract

Table 2: MIC of cefotaxime against Acinetobacter strains producing beta-lactamase

\begin{tabular}{llllllll}
\hline $\begin{array}{l}\text { Concentration of } \\
\text { cefotaxime }(\mu \mathrm{g} / \mathrm{ml})\end{array}$ & 25 & 50 & 100 & 200 & 300 & 400 & 500 \\
\hline
\end{tabular}

\begin{tabular}{llllllll}
\hline Number of strains inhibited & - & - & 7 & 4 & 2 & 8 & - \\
Percentage of strains inhibited & - & - & 33.3 & 19 & 9.5 & 38.0 & - \\
\hline
\end{tabular}

MIC: Minimum inhibitory concentration 
Table 3: Combined effect of cefotaxime and AGE Acinetobacter strains producing beta-lactamase

\begin{tabular}{lllll}
\hline Type of beta-lactamase & ESBL & AmpC & ESBL+AmpC & MBL \\
\hline Number of strains exhibiting synergy (FIC $\leq 0.5)$ & - & - & 10 & 1 \\
Number of strains exhibiting additive $(0.5<$ FIC $\leq 1)$ & - & - & 2 & 3 \\
Number of strains exhibiting indifference $(1<$ FIC $\leq 2)$ & - & 3 & - & 2 \\
Number of strains exhibiting antagonism (FIC $>2)$ & - & - & - & - \\
\hline
\end{tabular}

AGE: Aqueous garlic extract, ESBL: Extended-spectrum beta-lactamases, AmpC: Ambler Class C, MBL: Multiple beta-lactamase, FIC: Fractional inhibitory concentration

In the recent past, due to the high resistance of Acinetobacter species against beta-lactams, carbapenems had been the drugs of choice for serious infections with Acinetobacter [23]. However, carbapenemresistant strains are rapidly emerging; hence the study suggests a judicious usage of carbapenems in therapeutics.

The presence of beta-lactamase enzyme was confirmed based on phenotypic tests, and the results were in agreement with the study carried out by Singla et al. [8]. None of the isolates were pure ESBL producers. The production rate of $\mathrm{AmpC}$ alone and co-production rate of ESBL and AmpC beta-lactamases were in accordance with the study carried out by Singla et al. [8]. The prevalence rate of MBL producers in Acinetobacter species was well-corroborated with similar studies conducted in India $[24,25]$. Thus, in the current study, the principal mechanism of beta-lactam resistance was found to be co-expression of ESBL and AmpC beta-lactamases followed by MBL enzyme production. Incidences of co-expression beta-lactamases lead to erroneous treatment choices [26].

A significant observation of the current study was false susceptibility against piperacillin-tazobactam, in vitro by AmpC producers. This combination would presumably not be effective under in vivo conditions for AmpC producers. Similar false susceptibility was reported by Shinde et al. [27]. This suggests that the detection of AmpC should be performed routinely in hospitals. This would avoid therapeutic failures as it is a known fact that beta-lactam inhibitors are ineffective against AmpC producers under in vivo conditions.

The efficacy of many antibiotics in therapeutics has become quite limited owing to the development of resistance. The increasing failure of antibiotics has led to the screening of several plant extracts for their potential antimicrobial activity. If plant extracts are found to be effective, they could be explored further to overcome the problem of microbial drug resistance to antibiotics. Previous studies have shown that a wide range of microorganisms were sensitive to crushed garlic preparations, increasing the interest toward garlic as a medicinal panacea [14] The antimicrobial activity of garlic has been majorly attributed to its phytochemical component, allicin; a thiosulfonate. However, more studies are needed to be performed to determine the role of AGE to be used as an antibacterial agent against the beta-lactamase producers.

In the present study, all the test isolates were inhibited by AGE, and the results were comparable to the studies of Shahyan et al. [28] and Modi et al. [29]. Further, MIC of AGE was determined using the test isolates, and the results obtained were comparable with studies of Shahyan et al. [28]. This justifies the use of garlic in controlling bacterial infections since ancient days.

Synergy is one of the well-established indications for the successful combination of antimicrobial therapy. If a combination of a natural product and an antibiotic can cause the reversal of the antibiotic resistance or lower its dosage in therapy, this combination could potentially improve the outcome for patients with severe infections. Cefotaxime is the most preferable antibiotic prescribed during severe infections [30]. Hence, it was selected to study the synergistic interactions with AGE. The combination of AGE and cefotaxime showed at least a 4 -fold reduction in their respective MICs in $52.38 \%$ of the test isolates and a 2 -fold reduction in $23.8 \%$ of the test isolates. The antagonistic effect was not observed in any of the isolates, indicative of a positive association between the two antimicrobials. There is no documented study showing a synergistic effect between cefotaxime and AGE, but synergy and the partial synergistic association have been observed by allicin-cefoperazone combination [31].

To the best of our knowledge, no study has reported the effect of AGE against beta-lactamase-producing Acinetobacter strains from the SSTIs. The synergistic association between garlic and cefotaxime demonstrates the potential application of natural product and antibiotic combinations as an efficient, novel therapeutic tool for antibiotic-resistant bacterial infections.

\section{CONCLUSION}

Thus, it can be said that natural products have the ability to curb the growing menace of antibiotic-resistant bacteria. The use of different natural compounds as antibacterial agents is an interesting strategy for discovering bioactive products that could help in designing therapeutic tools. The augmented action of antibiotics along with natural substances may have positive synergistic effects toward specific, drug-resistant microorganisms, which are difficult to eradicate, particularly in hospital settings.

\section{ACKNOWLEDGMENT}

The authors are grateful to the Head of the Microbiology department and the Bacteriology section in-charge of Topiwala National Medical College and B.Y.L. Nair Charitable Hospital, Mumbai.

\section{REFERENCES}

1. Bergogne-Bérézin E, Towner KJ. Acinetobacter Spp. as nosocomial pathogens: Microbiological, clinical, and epidemiological features. Clin Microbiol Rev 1996;9(2):148-65.

2. Maragakis LL, Perl TM. Acinetobacter baumannii: Epidemiology, antimicrobial resistance, and treatment options. Clin Infect Dis 2008;46(8):1254-63.

3. Kwon NY, Kim JD, Pai HJ. The resistance mechanisms of b-lactam antimicrobials in clinical isolates of Acinetobacter baumannii. Korean J Intern Med 2002;17(2):94-9.

4. Fernandez-Cuenca F, Martinez-Martinez L, Conejo MC, Ayala JA, Perea EJ, Pascual A. Relationship between $\beta$-lactamase production, outer membrane protein and penicillin-binding protein profiles on the activity of carbapenems against clinical isolates of Acinetobacter baumannii. J Antimicrob Chemother 2003;51:565-74.

5. Thomson KS. Extended-spectrum-beta-lactamase, AmpC, and carbapenemase issues. J Clin Microbiol 2010;48(4):1019-25.

6. Kaur D, Puri J, Kulkarni S, Jayawant A. Prevalence of AmpC betalactamases in clinical isolates of $E$. coli from a tertiary care rural hospital. Int J Pharm Pharmacol Sci 2015;7(6):165-8.

7. Deepak S, Kamat S, Kamat D. Effect of aqueous extract of Terminalia chebula on metallobetalactamase. Int J Pharm Pharmacol Sci 2010;2(4):172-5.

8. Singla P, Sikka R, Deeep A, Gagneja D, Chaudhary U. Co-production of ESBL and AmpC B-lactamases in clinical isolates of A. baumannii and $A$. Lwoffii in a tertiary care hospital from Northern India. J Clin Diagn Res 2014;8(4):16-9.

9. Miyasaki Y, Nichols WS, Morgan MA, Kwan JA, Van Benschoten MM, Kittell PE, et al. Screening of herbal extracts against multi-drug resistant Acinetobacter baumannii. Phytother Res 2010;24(8):1202-6.

10. Nasim SA, Dhir B. Heavy metals alter the potency of medicinal plants. Rev Environ Contam Toxicol 2010;203:139-49.

11. Chowdhury AK, Ahsan M, Islam SN, Ahmed ZU. Efficacy of aqueous extract of garlic and allicin in experimental shigellosis in rabbits. Indian J Med Res 1991;93(1):33-6. 
12. Cavallito C, Bailey J. Allicin, the Antibacterial principle of Allium sativum. I. isolation, physical properties and antibacterial action. J Am Chem Soc 1944;66(11):1950-1.

13. Borlinghaus J, Albrecht F, Gruhlke MC, Nwachukwu ID Slusarenko AJ. Allicin: Chemistry and biological properties. Molecules 2014;19(8):12591-618.

14. Ankri S, Mirelman D. Antimicrobial properties of allicin from garlic. Microbes Infect 1999;1(2):125-9.

15. Colle JG, Miles RS, Watt B. Test for the identification of bacteria In: Collee JG, Faser AG, Marmion BP, Simmons A, editors. Practical Medical Microbiology. 14 ${ }^{\text {th }}$ ed. London: Churchill Livingstone; 1996. p. 131-45.

16. Clinical and Laboratory Standards Institute. Performance Standards for Antimicrobial Disc Susceptibility Tests: Approved Standard. $11^{\text {th }}$ ed. Wayne, PA: CLSI Document M02-A11. CLSI; 2012.

17. Shah P, Williamson M. Antibacterial and synergistic activity of Calendula officinalis methanolic petal extract on Klebsiella pneumoniae co-producing ESBL and AmpC beta-lactamase. Int J Curr Microbiol Appl Sci 2015;4(4):107-17.

18. Shah P, Williamson M. Antibacterial activity of honey against ESBL producing Klebsiella pneumoniae from burn wound infections. Int J Curr Pharm Res 2015;7(2):32-6.

19. Shah P, Williamson M, Rathod S. Antibacterial effect of methanolic neem leaf extract on ESBL, AmpC and MBL producers from skin and soft tissue infections. Int J Pharm Res Bio Sci 2016;5(3):117-28.

20. Eja M, Asikong B, Abriba C, Arikpo G, Anwan E, Enyi-Idoh K A comparative assessment of the antimicrobial effects of garlic (Allium sativum) and antibiotics on diarrhoeagenic organisms. Southeast Asian J Trop Med Public Health 2007;38:343-8

21. Nakamura A, Hosoda M, Kato T, Yamada Y, Itoh M, Kanazawa K, et al Combined effects of meropenem and aminoglycosides on Pseudomonas aeruginosa in vitro. J Antimicrob Chemother 2000;46(6):901-4.

22. Parandekar P, Peerapur P. Non-fermenters in human infections with special reference to Acinetobacter species in a tertiary care hospital from North Karnataka, India. J Krishna Inst Med Sci Univ 2012;1(1):84-8.

23. Mugnier P, Poirel L, Pitout M, Nordmann P. Carbapenem-resistant and OXA-23-producing Acinetobacter baumannii isolates in the united Arab Emirates. Clin Microbiol Infect 2008;14(9):879-82.

24. Shanthi M, Sekar U. Multi-drug resistant Pseudomonas aeruginosa and Acinetobacter baumannii infections among hospitalized patients: Risk factors and outcomes. J Assoc Physicians India 2009;57:636, 638-40.

25. Banerjee M, Chaudhary B, Shukla S. Prevalence of ESBL and MBL in Acinetobacter species isolated from clinical samples in tertiary care hospital. Int J Sci Res 2016;4(6):1183-6.

26. Pai H, Kang CI, Byeon JH, Lee KD, Park WB, Kim HB, et al. Epidemiology and clinical features of bloodstream infections caused by AmpC-type-beta-lactamase-producing Klebsiella pneumoniae. Antimicrob Agents Chemother 2004;48(10):3720-8.

27. Shinde S, Nataraj G, Mehta P. Multiple beta lactamase resistance mechanism in Escherichia coli and Klebsiella pneumoniae. Bombay Hosp J 2013;55(1):32-9.

28. Shayan S, Bokaeian M, Shahraki S, Saeidi, S. Prevalence of AmpC and ESBL producing E. coli and antibacterial effect of Allium sativum on clinical isolates collected from Zahedan hospitals. Zahedan J Res Med Sci 2014;16(4):6-10.

29. Modi K, Bhat A, Joshi P. Plasmid profiling of multidrug resistant isolates of E. coli, Klebsiella and Pseudomonas and antimicrobial potential of Allium sativum L. (garlic) against these isolates. Int J Curr Microbiol Appl Sci 2015;4(2):163-71.

30. Badar VA, Navale SB. Study of prescribing pattern of antimicrobia agents in medicine intensive care unit of a teaching hospital in central India. J Assoc Physicians India 2012;60(4):20-3.

31. Cai Y, Wang R, Pei F, Liang BB. Antibacterial activity of allicin alone and in combination with beta-lactams against Staphylococcus Spp. and Pseudomonas aeruginosa. J Antibiot (Tokyo) 2007;60(5):335-8. 